\title{
ESPACIOS ANTICIPATORIOS EN INFORMÁTICA \\ Dos propuestas educativas
}

\author{
Anticipatory Spaces in Informatics: Two Educational Proposals
}

\section{Viviana Harari, Claudia Mariana Banchoff TZancoff}

Laboratorio de Investigación en Nuevas Tecnologías Informáticas (LINTI), Universidad Nacional de La Plata (UNLP), Argentina

KEY WORDS

Anticipatory Space Institutional Affiliation Programming Teaching Free Software
ABSTRACT

The term "anticipatory space", used in multiple disciplines, had no formal definition in Informatics. Concepts related to the virtual were analysed in order to arrive at a definition within that field, and a possible classification was set forth. Moreover, FI3D is presented as an example, which is an application used as the basis for two educational proposals that allowed the analysis and evaluation of the impact of having an anticipatory space -- TIVU Virtual, a challenge-based serious game aimed at University first-year students, and ProBots3D, an environment for the first approach to programming at schools.
PALABRAS CLAVE

Espacio anticipatorio Afiliación institucional Enseñanza de programación Software libre
RESUMEN

El término "espacio anticipatorio", utilizado en varias disciplinas no contaba con una definición formal en Informática. Se analizaron conceptos relacionados con la virtualidad para llegar a dar una definición de ese término en esa área, y se propuso una posible clasificación del mismo. Además, a modo de ejemplo se presenta FI3D, aplicación usada como base de dos propuestas educativas que permitieron analizar y evaluar el impacto de contar con un espacio anticipatorio: TIVU Virtual, un juego serio basado en desafíos destinado a ingresantes a la universidad y ProBots3D, un entorno para realizar las primeras actividades prácticas de programación en escuelas. 


\section{Introducción}

$\mathrm{L}$ a Facultad de Informática de la Universidad Nacional de La Plata (UNLP) tiene un ingreso promedio de 800 estudiantes por año ${ }^{1}$. En esta unidad académica se dictan carreras muy demandadas tanto en la región como en otras regiones del país y de América Latina. Muchos estudiantes, principalmente los que provienen de regiones más lejanas, desconocen la zona en donde está situada la facultad y las instalaciones propias que la misma ofrece. Poder "ver" el lugar antes de concurrir al mismo, genera menos incertidumbre al momento de concurrir al lugar físico real (Harari V. \& Banchoff C., 2016). Para situar a la facultad geográficamente y visualizar sus alrededores, basta con consultar cualquier mapa en Internet, como pueden ser OpenStreetMap ${ }^{2}$ o Google Maps ${ }^{3}$. Para complementar y extender la información que otorga un mapa geográfico se desarrolló un modelo 3D representativo de la facultad, al cual se lo denominó FI3D: Facultad de Informática en 3D. Dado que este desarrollo se enmarca en un proyecto de investigación se dió un marco teórico y se definió el concepto de "espacio anticipatorio" en términos informáticos.

En la sección 2 de este artículo se describe el concepto de espacio anticipatorio en términos informáticos, se establece su alcance, se da una clasificación y se presenta a la aplicación FI3D como un ejemplo de dicho concepto.

En la sección 3 se describen dos propuestas surgidas en base a FI3D y en la sección 4 se analiza el impacto de su uso como espacio anticipatorio.

\section{Espacio anticipatorio}

En el año 2014, en el marco de un proyecto del Laboratorio de Investigación en Nuevas Tecnologías Informáticas (LINTI) ${ }^{4}$, se comienza con el desarrollo de FI3D, un espacio virtual 3D, que se

1 Anuario Estadístico 2017: indicadores comparados: http://www.unlp.edu.ar/indicadores. Último acceso: septiembre 2018.

2 La Facultad de Informática en OpenStreetMap: https://goo.gl/8QeYAb. Último acceso: septiembre 2018.

3 La Facultad de Informática en Google Maps: https://goo.gl/AQT4nD. Último acceso: septiembre 2018.

4 Sitio ofifial del Laboratorio de Investigación de Nuevas Tecnologías Informáticas (LINTI): https://www.linti.unlp.edu.ar/. Último acceso: septiembre 2018. presenta como un "espacio anticipatorio". Este desarrollo tuvo como objetivo principal construir una herramienta que permitiera sensibilizar y familiarizar respecto a la estructura edilicia de la facultad. Si bien se trata de una herramienta que puede ser utilizada por cualquier persona que quiera conocer la entidad, en sus inicios estuvo pensada básicamente para los estudiantes ingresantes a la facultad, de manera tal de disminuir la incertidumbre, duda o temor que provoca la llegada a un lugar nuevo y desconocido.

El conocer previa y virtualmente un lugar permite que, a la hora de realizar la visita real, la persona sienta que ese espacio le es familiar y conocido, como si hubiese estado allí anteriormente.

Si bien el término "espacio anticipatorio" es un concepto utilizado, en varias áreas como lo son la medicina o la psicología, para referirse a recursos que sensibilizan y familiarizan a las personas sobre procesos o procedimientos (Croci de Romero L. \& González S., 2017), en el área de la Informática, no es tan habitual su uso. Es por este motivo que, frente al desarrollo planteado, se decidió dar un marco teórico y una definición formal a este concepto, en esta área.

Partiendo de la definición primaria sobre espacio anticipatorio, que lo asocia con un "espacio virtual 3D", se llega a la definición formal del mismo: un espacio anticipatorio es "un ambiente virtual $3 D$ representativo de un espacio físico particular, creado y diseñado con el propósito de permitir su acceso para recorrerlo, conocerlo y sensibilizarse con el lugar." (Harari V., 2018).

Para alcanzar esta definición se analizaron en forma exhaustiva los conceptos informáticos relacionados con la virtualidad: realidad virtual, mundo virtual y entorno y ambiente virtual.

En una primera instancia, analizando las múltiples definiciones sobre realidad virtual, se toma la dada por la Facultat d'Informàtica de Barcelona que plantea que la realidad virtual

Es un sistema informático que genera en tiempo real representaciones de la realidad, que de hecho no son más que ilusiones ya que se trata de una realidad perceptiva sin ningún soporte físico y que únicamente se da en el interior de los ordenadores". (Facultat d'Informàtica de Barcelona, 2018). 
De esta manera se concluye que el concepto de espacio anticipatorio se puede definir en término de realidad virtual, por tener características que responden a las que tienen estos sistemas.

Siguiendo con la investigación se analizan los términos de entorno y ambiente virtual con el objetivo de establecer si ambos términos son equivalentes, e indagar sobre su relación con el concepto de espacio anticipatorio. En esta línea se toma como referencia el trabajo de Diego Leal (Leal D., 2011), en el cual se plantea si la traducción del término en inglés enviroment, corresponde a ambiente o a entorno y, si ambos términos son equivalentes. Leal concluye que estos términos son diferentes ya que la palabra entorno hace referencia a "lo que rodea a algo $o$ alguien", mientras que la palabra ambiente

... deriva de la interacción del hombre con el entorno natural que lo rodea. Se trata de una concepción activa que involucra al ser humano $\mathrm{y}$, por tanto, involucra acciones pedagógicas en las que quienes aprenden están en condiciones de reflexionar sobre su propia acción y sobre las de otros (Duarte citado por Leal D., 2011, p.146).

Teniendo en cuenta esta diferenciación, el concepto de espacio anticipatorio se enmarca también en ambiente virtual.

Finalmente cuando se indaga sobre mundos virtuales respecto a sus características, tipologías y clasificación, se concluye que un espacio anticipatorio sólo se lo considera como un mundo virtual si incluye características y funcionalidades tales como: funcionar persistentemente independientemente si los usuarios están conectados o no; utilizar avatares para representar a los usuarios; incluir interactividad en forma remota y en simultáneo; permitir múltiples usuarios conectados; entre otras cosas.

\subsection{Clasificación propuesta}

Una vez dado el marco teórico del concepto de espacios anticipatorios, se avanza sobre una propuesta de clasificación de los mismos. Para esto se analizaron características tales como: persistencia, nivel de inmersión, interacción y cantidad de usuarios conectados en forma simultánea.
La persistencia hace referencia al funcionamiento del sistema, independientemente si el usuario se encuentra conectado o no. El nivel de inmersión indica el grado de involucramiento de los sentidos de los usuarios. Cuantos más dispositivos tales como cascos, lentes, entre otros, se utilicen, más involucrado tendrá sus sentidos el usuario y, sentirá aún más la sensación de estar dentro de esa realidad virtual. En lo que a interacción del usuario con el espacio se refiere, se puede plantear tres niveles de interacción (Mejía Luna J., 2012, p.25):

- Explorativa: el usuario no puede modificar ninguna parte $\mathrm{u}$ objeto del entorno, sólo puede recorrer y visualizar los elementos del mismo.

- Manipulativa: el usuario puede mover, rotar y escalar los objetos, pero esto no modifica el entorno virtual en esencia, tan solo se consigue una reconfiguración posicional de los objetos.

- Contributiva: el usuario puede realizar cambios a nivel del estado funcional o reactivo, es decir los objetos del espacio pueden modificar su aspecto además de su posición, se puede agregar o eliminar objetos.

En base a lo planteado se propuso, en una primera instancia, dos niveles de espacios anticipatorios con las siguientes particularidades:

- Espacio anticipatorio básico o de nivel 1: es aquel que no es persistente, presenta un nivel de inmersión bajo ${ }^{5} y$ tiene, por parte de un único usuario, una interacción explorativa o simple. El usuario, con su accionar, no produce cambios en el ambiente.

- Espacio anticipatorio de nivel 2: es aquel que no es persistente, también presenta un nivel de inmersión bajo y tiene, por parte de un único usuario, una interacción contributiva. Por lo tanto el usuario, con su accionar, puede producir algunos cambios en el ambiente.

Retomando la definición formal de espacios anticipatorios se puede extender la misma a una definición más completa:

\footnotetext{
${ }^{5}$ Se considera un nivel de inmersión bajo cuando el usuario no cuenta con dispositivos de realidad virtual.
} 
Un espacio anticipatorio es un ambiente virtual 3D representativo de un espacio físico particular, creado y diseñado con el propósito de permitir su acceso para recorrerlo, conocerlo y sensibilizarse con el lugar. Tiene como objetivo anticiparse al recorrido real. Se enmarca dentro de la realidad virtual y puede clasificarse en diferentes niveles de acuerdo al grado de interacción, nivel de inmersión, cantidad de usuarios conectados en forma simultánea y persistencia que presente. (Harari V., 2018).

\subsection{FI3D: una implementación de un espacio anticipatorio}

Como se mencionó en secciones anteriores, FI3D es un software que permite realizar un recorrido virtual por las instalaciones de la Facultad de Informática de la UNLP. A través de un avatar, el usuario puede visualizar las aulas, las oficinas administrativas, la biblioteca y el buffet, entre otros espacios.

El desarrollo de FI3D fue evolucionando a lo largo del tiempo. La primera versión, denominada FI3D 1.0 se enmarca en lo que se definió como espacio anticipatorio básico o de nivel 1, dado que es mono-usuario, la única interacción del usuario con el ambiente es el desplazamiento por el lugar y no tiene posibilidad de intervenir ni modificar el ambiente.

La figura 1 muestra algunas de las capturas de la aplicación FI3D 1.0 desarrollada. En la misma pueden observarse dos espacio físicos reales de facultad y su correspondiente representación virtual.

Figura 1. Vistas de espacios físicos de la facultad y su representación en FI3D.

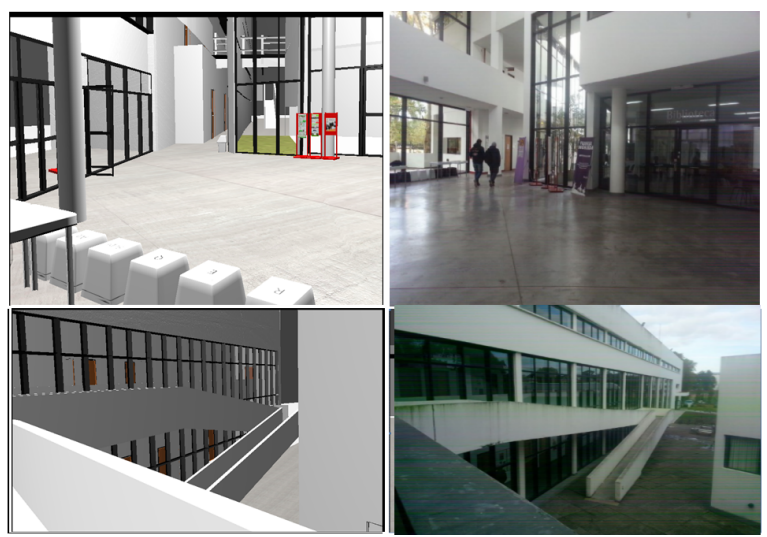

Fuente(s): elaboración propia, año 2017.
La segunda versión, denominada FI3D 2.0, incorpora modificaciones tanto a nivel edilicio como a nivel funcional permitiendo enmarcarla en el concepto de espacio anticipatorio de nivel 2. Se trata de una aplicación mono-usuario, pero en esta versión la interacción con el ambiente, además de poder desplazarse por el lugar, el usuario puede intervenir en el mismo.

Si bien fueron cambios significativos los que se realizaron, el más importante fue el de permitir agregar información al ambiente, y asociarla a distintos objetos tales como las diferentes puertas de las dependencias de la entidad.

La figura 2 muestra alguno de los cambios realizados.

Figura 2. Diferencias entre FI3D 1.0 y FI3D 2.0. Espacio anticipatorio de nivel 1 y 2 .

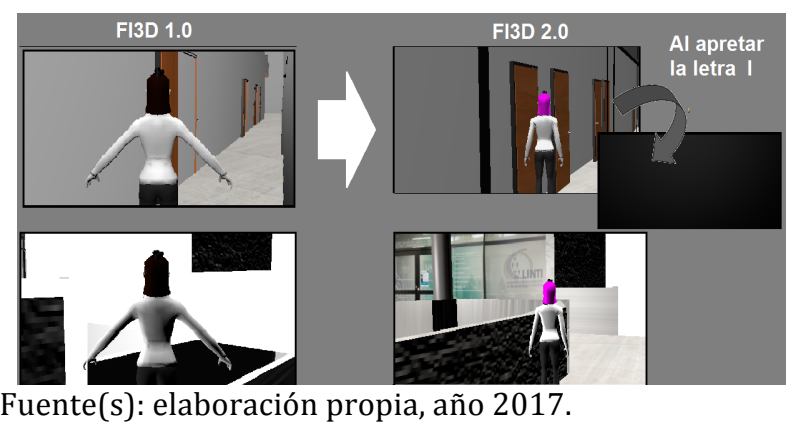

\subsection{Detalles de implementación}

FI3D es un desarrollo de software libre, donde tanto los modelos involucrados como las herramientas de desarrollo y el producto final son libres ${ }^{6}$. Esto permite que no sólo pueda utilizarse por quienes así lo deseen, sino que puede modificarse y adaptarse a diferentes necesidades.

Luego de analizar varias herramientas se decidió trabajar con Blender ${ }^{7}$ para el desarrollo del primer prototipo. Si bien en un primer momento sólo se pensó en utilizar esta herramienta para el modelado de los recursos necesarios, luego se decidió continuar con el desarrollo del software manteniendo el mismo entorno.

6 La definición de software lbre puede encontrarse en https://www.gnu.org/philosophy/free-sw.es.html. Último acceso septiembre 2018.

${ }^{7}$ Sitio oficial: https://www.blender.org/. Último acceso septiembre 2018. 


\section{Dos propuestas educativas basadas en FI3D}

Frente a dos propuestas educativas que, en un principio estaban destinadas a estudiantes de nivel secundario, surgió como idea interesante desarrollarlas en base a FI3D. De esta manera se pudo probar el concepto de espacio anticipatorio y comprobar, de forma empírica, las ventajas de su uso.

Ambas propuestas surgieron como trabajos de tesis de la maestría en Tecnología Informática Aplicada en Educación de la Facultad de Informática de la UNLP.

La primera de ellas, denominada TIVU Virtual, propone un juego serio basado en desafíos destinado a futuros ingresantes a la facultad. ProBots3D, la otra propuesta, se integra al proyecto "Programando con robots y software libre" (Dïaz J., Banchoff Tzancoff C. Queiruga C. \& Martin E. 2014). y brinda un entorno para realizar las primeras actividades prácticas de programación en dicho proyecto.

\subsection{TIVU Virtual}

El ingreso a la universidad requiere, por parte de los estudiantes ingresantes, adaptaciones al nuevo ámbito. Esto implica una reorganización personal, familiar, social y, una nueva manera de relacionarse con el conocimiento. Estos procesos a los que Jesús E. Valenzuela (Valenzuela, 2011), denomina "procesos de integración", y Alan Coulon (Coulon, 1995, 2005) denomina proceso de "afiliación", son necesarios para que el ingresante alcance el aprendizaje del "oficio de estudiante".

Este proceso de afiliación o integración se plantea tanto a nivel académico como institucional y social. Esto significa que el estudiante debe aprender a relacionarse con la disciplina que va a estudiar, aprendiendo "a leer y escribir" en ella. Debe también aprender sobre la cultura administrativa del nuevo ámbito educativo en el cual comienza a incursionar, conociendo sus normas, reglas, organización, entre otros $\mathrm{y}$, debe también aprender a relacionarse con otros actores como lo son los docentes, administrativos, técnicos, directivos y pares de otras cohortes.
Las entidades educativas universitarias, en su mayoría, dedican tiempo y esfuerzo para encontrar, aplicar y mejorar mecanismos y procesos que permitan facilitar la integración de los nuevos estudiantes.

En particular la Facultad de Informática de la UNLP, implementa un curso de ingreso para las carreras que dicta: Licenciatura en Informática, Licenciatura en Sistemas, Analista Programador Universitario (APU) y Analista en Tecnologías de la Información y la Comunicación (ATIC). La facultad también dicta, en forma conjunta con la Facultad de Ingeniería de la UNLP, la carrera de Ingeniería en Computación para la cual implementa un curso llamado "Curso de Nivelación". La organización de este curso está a cargo de la Facultad de Ingeniería.

El curso de ingreso que dicta la Facultad de Informática está relacionado con la integración académica y, para el año 2019, propuso tres módulos disciplinares: "Expresión de Problemas y Algoritmos", "Conceptos de Organización de Computadoras" y "Matemática 0". Este curso también está acompañado de un taller denominado "Taller de Inserción a la Vida Universitaria (TIVU)". El mismo está relacionado con la integración institucional y social ya que tiene como objetivo brindar información a los nuevos estudiantes sobre la historia de la institución, su estructura y funcionamiento, los servicios y becas que ofrece la universidad, los derechos de los estudiantes, la participación estudiantil y la importancia de la misma, entre otras cosas. De esta manera la Facultad de Informática año a año pone en juego estos mecanismos que ayudan a la integración o a la afiliación de sus nuevos estudiantes.

TIVU Virtual es una propuesta de un juego serio educativo que fue pensado como un complemento del taller TIVU. Se trata de un juego basado en un espacio anticipatorio de nivel 2 que tiene como objetivo que los usuarios, futuros ingresantes a la facultad, puedan familiarizarse o sensibilizarse con la entidad. Permite también que los mismos puedan identificar y localizar oficinas, aulas, lugares de recreación, entre otros $\mathrm{y}$, conocer los trámites administrativos que se realizan en las diferentes dependencias, junto con la documentación a presentar en cada caso. 
Propone dos modos de uso que permiten aprender y sensibilizarse con el lugar. En uno de ellos, al que se denominó "Recorrer", el usuario pueda transitar por la institución para sensibilizarse con el lugar, aprendiendo sobre algunos de los trámites administrativos que se realizan en la misma. El otro modo de uso, denominado "Jugar", permite que el usuario se auto-evalúe realizando un desafío planteado por la aplicación.

La definición completa de TIVU Virtual quedó definida en el marco de la tesis de Magister "Taller virtual de inserción a la vida universitaria: Espacio anticipatorio educativo basado en un juego de desafíos con herramientas libres" (Harari V., 2018).

Para evaluar el impacto de uso de una herramienta con estas características, sobre todo en lo que concierne a su funcionalidad como espacio anticipatorio, se implementó una aplicación básica basada en la propuesta original del juego.

Para llevar a cabo este desarrollo se partió de FI3D 2.0, que, como se mencionó anteriormente, es un espacio anticipatorio de nivel 2.

En la figura 3 se puede observar una captura del juego en los dos modos de uso. En el modo "Recorrer" el usuario se encuentra con un cartel de bienvenida, mientras que en el modo "Jugar", en dicho cartel se presenta el desafío a resolver.

Figura 3. TIVU Virtual 1.0 en modo "Recorrer" y "Jugar"

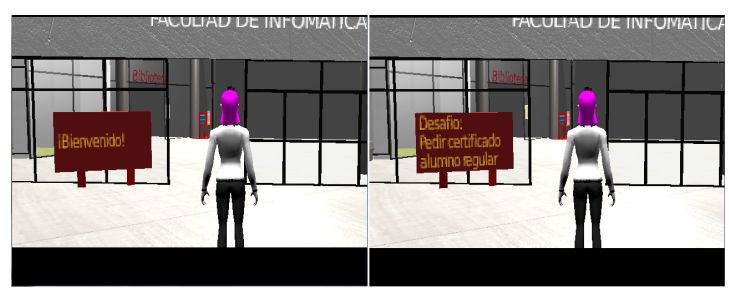

Fuente(s): elaboración propia, año 2017.

En la aplicación, algunas de las dependencias presentan carteles con una marca que indican que se puede interactuar con dicha dependencia. Si se está en modo "Recorrer", al interactuar con este sitio se muestra un cartel con información sobre el lugar y los trámites que se realizan allí. Si se está en modo "Jugar", se indica que dicha dependencia es uno de los lugares involucrados en el trámite planteado por el desafío.
La figura 4 muestra la forma en que los diferentes carteles se presentan en cada modo.

\section{Figura 4. TIVU Virtual 1.0 carteles en modo "Recorrer" y "Jugar"}

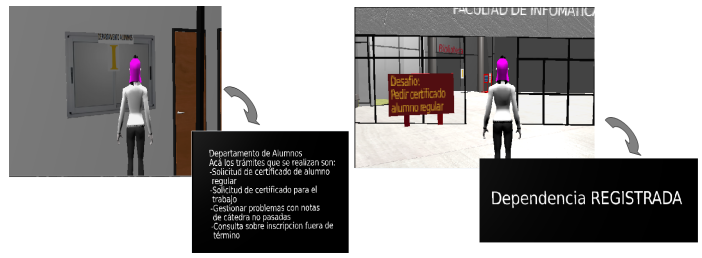

Fuente(s): elaboración propia , año 2017.

\subsection{ProBots3D}

Desde hace más de una década se viene trabajando en distintas formas de incluir las TIC en la dinámica del aula de la escuela y, más específicamente, el abordaje de la Informática como una ciencia, siendo la programación de computadoras una parte esencial de la misma.

Aprender a programar permite que los estudiantes asuman un rol activo en relación al uso de las computadoras y los elementos tecnológicos que los rodean. De esta manera, ellos pueden proponer soluciones creativas y divergentes, donde se ponen en juego su aporte personal.

El proyecto "Programando con robots y software libre", llevado a cabo por docentes e investigadores del LINTI, trabaja en esta línea. Su objetivo principal consiste en abordar los conceptos básicos de la programación de computadoras a través del uso de pequeños robots inalámbricos que se programan en el lenguaje Python. En el marco de este proyecto se desarrollaron varias herramientas para complementar el uso de los robots en diferentes contextos y para diferentes destinatarios. Entre ellas, se cuenta con dos aplicaciones denominadas DuinoBotSocks ${ }^{8}$ y Dropsy $^{9}$, las cuales permiten introducir las actividades iniciales con los robots tanto en niños pequeños como en jóvenes adolescentes a través de una

\footnotetext{
${ }^{8}$ DuinoBotSocks fue desarrollada por integrantes deI LINTI. y puede descargarse desde https://github.com/RobotsLinti/DuinoBotSocks. Último acceso, octubre de 2018.

${ }^{9}$ Dropsy surge como el trabajo de grado de los alumnos Matías Fuentes y Diego Fernández. El mismo puede descargarse desde http://sedici.unlp.edu.ar/handle/10915/67010. Último acceso, octubre de 2018.
} 
interfaz icónica o basada en bloques respectivamente, para luego pasar al lenguaje Python. Aunque el uso de ambas herramientas resulta positivo como actividad introductoria, el inconveniente planteado en ambos casos es la necesidad de contar con los robots físicamente, dado que estas aplicaciones se conectan al robot a través de una conexión WIFI.

Al evaluar los buenos resultados que se obtuvieron al introducir la programación con una interfaz más amigable, se propuso incorporar una nueva herramienta que no requiera de los robots físicos para estas primeras actividades. Como en el proyecto "Programando con robots y software libre" participan tanto estudiantes de nivel primario como secundario, se pensó en desarrollar esta nueva herramienta basada en FI3D. Esto permite, entre otras cosas, promover a la Facultad de Informática como un lugar para continuar los estudios. Surge así ProBots3D, que no solamente permite trabajar los aspectos de programación básicos relacionados al proyecto, sino que también permite familiarizarse con las instalaciones de la facultad, proponiendo a ProBots3D como un "espacio anticipatorio" (Harari et al, 2016).

ProBots3D se basó en la primera versión de FI3D, a la cual se le agregó un conjunto de robots dispersos en el espacio de la facultad. Al aproximarse a cada uno de ellos, se muestra un desafío de programación. La figura 5 muestra una captura de ProBots3D en donde se puede visualizar un robot y uno de los desafíos asociados.

Figura 5. Captura del hall de entrada en ProBots3D y el desafío asociado al robot presente en el mismo.

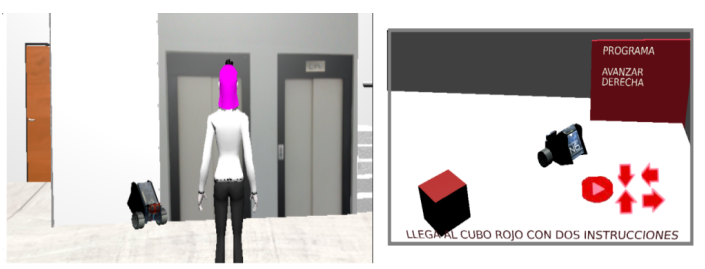

Fuente(s): elaboración propia, año 2017.

ProBots3D presenta dos versiones: una destinada a estudiantes de nivel primario y otra para estudiantes de nivel medio. En ambas versiones el escenario y el modo de uso es similar. La consigna es localizar los robots dispersos en el edificio de la Facultad de Informática y resolver el desafío de programación planteado a través de una interfaz icónica. En una pizarra virtual se muestra el programa generado por los estudiantes que, para el caso del nivel primario, el mismo se muestra en lenguaje natural. En el caso de la versión para el nivel medio, el programa se muestra en lenguaje Pyrhon, como una forma de familiarizar a los estudiantes con dicho lenguaje. La descripción completa de ProBots3D y los detalles de su uso en un marco pedagógico está disponible en (Banchoff Tzancoff, 2018).

\section{Las experiencias realizadas}

Tanto TIVU Virtual como ProBots3D fueron evaluadas de manera tal de analizar y corroborar el impacto de las mismas en los contextos en los cuales estaban inmersas. Pero en ambos casos también se las evaluó como espacios anticipatorios. En esta sección se detalla cada una de las evaluaciones realizadas.

\subsection{TIVU Virtual}

TIVU Virtual se probó con dos grupos de personas de perfiles muy diferentes. Uno de esos grupos estuvo conformado por jóvenes que asisten a un comedor barrial llamado Centro Verde Esperanza ${ }^{10}$, localizado en el barrio Jardín de alrededores de la ciudad de La Plata, provincia de Buenos Aires. Esta institución forma parte del grupo de entidades participantes del proyecto de extensión y voluntariado "El Barrio va a la Universidad", de la Facultad de Informática (Díaz J., Harari I. \& Harari V., 2013). Estos jóvenes asisten a escuelas públicas de la zona y, diariamente van al comedor barrial a recibir apoyo escolar y una copa de leche. El otro grupo estuvo conformado por profesionales, estudiantes universitarios y ex alumnos de escuelas secundarias privadas o pertenecientes a la UNLP. En ambos grupos, los participantes solamente tenían un conocimiento básico sobre el uso de la computadora.

Dado que TIVU Virtual fue pensada para ser utilizada como complemento de un taller de ingreso a una facultad que pertenece a una

${ }^{10}$ Sitio oficial: http://fundaciontau.org.ar/. Último acceso, octubre de 2018. 
universidad nacional pública y gratuita, pareció importante contar con todas las opiniones. Esto refiere a personas que tienen o han tenido diferentes posibilidades educativas.

En ambos grupos se realizaron dos encuestas, una antes de utilizar el software y otra al finalizar la prueba del mismo.

La primer encuesta sirvió para tener un perfil de los participantes de acuerdo a diferentes cuestiones como por ejemplo: conocimiento respecto a trámites administrativos universitarios, conocimiento respecto al manejo de la computadora, utilización de juegos con avatares, entre otros.

Los resultados mostraron que todos los participantes usaban la computadora para, al menos, buscar información y estudiar. Respecto al uso de juegos con avatares, el 85\% no había utilizado juegos con esas características. Respecto a trámites administrativos, todos los adultos manifestaron tener conocimiento sobre el tema pero el $85,71 \%$ de los jóvenes no.

En cuanto a la segunda encuesta, que se realizó una vez probada la aplicación, los resultados arrojaron los siguientes:

- A todos los participantes les gustó la aplicación y el hecho de estar representado por un avatar.

- A todos los adultos les dió la sensación de estar dentro de la facultad y pensaron que, si fueran realmente a la misma, reconocerían sus lugares. En el caso de los jóvenes, el 85,71\% expresó lo mismo.

- En general, a todos los participantes les resultó sencillo la consigna planteada. El $57 \%$ de los jóvenes resolvieron el desafío en un solo intento y en el caso de los adultos el 66\%. El resto lo resolvió con al menos dos intentos.

Si bien estos resultados parecen parejos entre estos dos grupos, un dato significativo se dió cuando se preguntó respecto a proponer alguna modificación o cambio al software, En el caso de los adultos el $83 \%$ propuso algunos cambios, algunos de ellos muy importantes. En el caso de los jóvenes el 85,71\% no propusieron cambios.

En todos los casos, durante las pruebas además se llevó una bitácora o diario de campo que permitió registrar observaciones sobre la experiencia. Sólo se asistió a los participantes en el caso que lo solicitaron explícitamente.

La aplicación también fué sometida a una evaluación de expertos. Para esto se solicitó a la Lic. Ana Úngaro, directora de la Dirección de Orientación al Alumno de la Facultad de Informática de la UNLP y encargada de la organización del taller TIVU, que observe la aplicación y realice las devoluciones correspondientes. Las principales opiniones recibidas por parte de la profesional fueron positivas, sobre todo en lo que respecta al uso de la herramienta como complemento del taller, con especial atención a la posibilidad de su inclusión en la modalidad virtual del mismo. La profesional también remarcó la importancia de la aplicación como una herramienta complementaria para sensibilizar, aprender o repasar.

\subsection{ProBots3D en dos escuelas}

ProBots3D fue utilizada con dos grupos de estudiantes correspondientes a nivel primario y secundario de dos escuelas de la ciudad de City Bell, provincia de Buenos Aires (Argentina): la escuela primaria 67 "Enrique Galli" y la escuela "Estudiantes de La Plata". En ambos grupos de estudiantes, se relevó información sobre los conocimientos previos, los datos de la interacción con la herramienta y otra información de interés. En el caso de los estudiantes de nivel secundario, este relevamiento se realizó a través de tres encuestas que fueron completadas directamente por los mismos estudiantes, mientras que en el caso del nivel primario, el relevamiento se realizó a través de una breve entrevista.

Si bien la prueba de campo se focalizó en el objetivo propio de la herramienta (trabajar aspectos de programación), también se evaluó su uso como espacio anticipatorio. Sobre esto último, la evaluación brindó datos significativos en los estudiantes de nivel secundario que conocían o luego conocieron las instalaciones de la facultad.

De todos los estudiantes secundarios evaluados, solamente uno de ellos conocía la facultad y al trabajar con el software pudo reconocer los distintos espacios. Luego de la prueba, el $75 \%$ de ellos concurrieron a la facultad para una actividad en el marco del proyecto y el $90 \%$ de los mismos pudieron 
ubicar los distintos espacios visualizados en el software. Esto permitió encarar las actividades en un lugar que les resultaba familiar.

\subsection{Resultados generales de ambas pruebas}

Teniendo en cuenta las evaluaciones realizadas con ambas herramientas se pueden mencionar algunas conclusiones generales:

- En el momento de utilizar ambas aplicaciones los estudiantes mostraron un gran interés en recorrer el espacio y visitar las instalaciones teniendo en cuenta que se trataba del lugar de trabajo de las docentes a cargo de la prueba.

- Algunos participantes propusieron incorporar un mapa que indique la ubicación contextual del avatar en el espacio.

- En ambos casos, también solicitaron más desafíos a resolver.

- En el caso de TIVU Virtual les pareció interesante incorporar otros usuarios interactuando en el mismo ambiente.

- Aproximadamente el $80 \%$ de los participantes que no conocían la facultad, al concurrir luego a la misma, pudieron ubicarse más fácilmente y reconocer sectores tales como el buffet, biblioteca y las salas de PC.

\section{Conclusiones}

Conocer previamente, aunque sea en forma virtual, un lugar antes de concurrir al mismo, permite que, al hacerlo en forma real, se llegue familiarizado con el espacio, evitando la sensación de ansiedad e inseguridad que provoca el arribo a un lugar nuevo. Esto se enfatiza aún más cuando va acompañado de cambios más profundos como puede ser el ingreso a la universidad.

Este trabajo permitió analizar y evaluar el impacto que produce contar con un espacio anticipatorio representativo de la Facultad de Informática de la UNLP en potenciales ingresantes a la misma.

Las experiencias realizadas también permitieron mostrar a la universidad como un espacio posible para continuar los estudios $y$, en particular, la disciplina informática.

Un aspecto a destacar también en este trabajo es que ambos desarrollos pudieron realizarse gracias a que la aplicación utilizada como base fue desarrollada como software libre. Esto permite que quienes así lo deseen puedan utilizar y/o actualizar los desarrollos. 


\section{Referencias}

Banchoff Tzancoff, C. (2018). ProBots3D: programando Robots en 3D. Una herramienta libre para enseñar programación a niños y jóvenes. Disponible en http://hdl.handle.net/10915/67659 Último acceso julio de 2020.

Croci de Romero, L. \& González, S. (2017) "Psicoprofilaxis de la revisación: Abriendo puertas para un abordaje integral". Disponible en: http://www.buenosaires.gob.ar/areas/salud/dircap/mat/trabajos/psicoped/07/laxis.pdf. Último acceso julio de 2020.

Coulon, A. (1995). Etnometodología y educación. Barcelona. Ed. Paidos.

- (2005). Le métier d'étudiant. L'entrée dans la vie universitaire. Paris. Ed. Deuxième.

Dïaz, J., Banchoff Tzancoff, C. Queiruga, C. \& Martin, E. (2014). Experiencias de la Facultad de Informática en la Enseñanza de Programación en Escuelas con Software Libre. Congreso Iberoamericano de Ciencia, Tecnología, Innovación y Educación. Buenos Aires, Argentina.

Diaz J, Harari, I. y Harari V. (2013). University project: The marginalized neighborhood goes to University .Marrakech, Marruecos. ISBN 978-9954-9091-2-6.

Facultat d'Informàtica de Barcelona (2018) . Realidad virtual. Disponible en https://www.fib.upc.edu/retro-informatica/avui/realitatvirtual.html Último acceso: julio de 2020.

Harari V. \& Banchoff C. (2016). FI3D: An Anticipatory Space. International Conference on Engineering Education \& Research ICEER. Australia. ISBN: 978-0-646-95724-1, 154-160.

Harari, V. (2018). Taller virtual de inserción a la vida universitaria (Doctoral dissertation, Facultad de Informática). Disponible en http://hdl.handle.net/10915/67661. Último acceso julio de 2020.

Leal, D. (2011). Ambientes personales de aprendizaje en el desarrollo profesional docente. Capítulo VI del libro "El modelo Ceibal: Nuevas tendencias para el aprendizaje". Página 146-147 .

Mejía Luna, J. N. (2012). Realidad Virtual, Estado del arte análisis crítico (Master's thesis, Universidad de Granada/2012).

Valenzuela Medina J. E., (2011). Formación De Identidad En Estudiantes Universitarios: Un Diseño Combinado Secuencial Explicativo. Tesis Doctoral. Disponible en: https://www.ciad.mx/archivos/desarrollo/publicaciones/Tesis\%20asesoradas/Doctorado/Valen zuelaErnesto.pdf. Último acceso julio de 2020. 